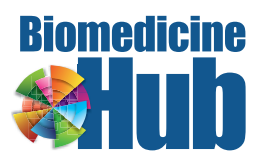

\title{
Nasal Cavity Paraganglioma: Literature Review and Discussion of a Rare Case
}

\author{
Juliana Maria de Almeida Vital ${ }^{a}$ Terence Pires de Farias ${ }^{b, c}$ Fernando Luiz Dias ${ }^{a, c, d}$ \\ Juliana Fernandes de Oliveira ${ }^{a}$ José Gabriel Miranda da Paixão ${ }^{a}$ \\ Paulo José de Cavalcanti Siebra ${ }^{a}$ Arli Regina Lopes Moraes ${ }^{a}$ \\ ${ }^{a}$ Department of Head and Neck Surgery and bepartment of Oncology, Instituto Nacional de Câncer \\ (INCA), and ${ }^{\mathrm{C}}$ Department of Head and Neck Surgery, Pontíficia Universidade Católica of Rio de \\ Janeiro, Rio de Janeiro, and ${ }^{\mathrm{d}}$ Department of Clinical Surgery, Universidade de São Paulo, \\ São Paulo, Brazil
}

\section{What Is It about?}

Paragangliomas can be found from the skull base to the sacrum. This article provides a review of sinonasal paraganglioma, which is infrequent and mainly occurs in middle-aged women, and reports on an illustrative case. Radiologic investigation is essential for the diagnosis of sinonasal paraganglioma and these must be resected, aiming for free margins, using either an endonasal or conventional approach with long-term follow-up.

\section{Keywords}

Paraganglioma $\cdot$ Sinonasal paraganglioma $\cdot$ Nasal cavity paraganglioma

\begin{abstract}
Paragangliomas can be found from the skull base to the sacrum. Sinonasal paragangliomas are infrequent. A 16-year-old female reported spontaneous discrete bilateral epistaxis once a month beginning when she was 3 years of age. Computed tomography showed an expansive hypervascular mass occupying the right nasal cavity and nasopharynx. Sinonasal paragangliomas usually occur in middle-aged women. Radiologic investigation is essential for the diagnosis of sinonasal paragangliomas and evaluating extension of the lesion. Endoscopic and conventional approaches are effective, and preoperative embolization is paramount for reducing bleeding risk. Histopathological features cannot differentiate benign from malignant paragangliomas, and since metastasis may eventually occur, follow-up must be carried out for a long period of time.




\section{Established Facts}

- Paragangliomas are neuroendocrine tumors which can be found from the skull base to the sacrum. Sinonasal paragangliomas are infrequent [Tolman and Stam: BJR Case Reports 2016;2016005].

- Their histological features are not accepted as definitions of malignancy criteria. Malignant paragangliomas are defined by lymph node or distant metastasis and may occur in about $10 \%$ of patients [Pellitteri: Oral Oncol 2004;40:563-575; Taïeb et al.: J Nucl Med 2012;53:264-274].

- Diagnostic investigation includes catecholamine excess evaluation, computed tomography, and magnetic resonance imaging. Nuclear imaging may be required for secondary lesion investigation. Genetic mutations in the succinate dehydrogenase genes have been described in paraganglioma patients, and those with early onset should be evaluated when genetic testing is available.

- Sinonasal paragangliomas must be resected, aiming for free margins, either by an endonasal or by conventional approach [Ketabchi et al.: Eur Arch Otorhinolaryngol 2003;260:336-340].

- Radiation therapy may control the disease by slowing its growth rate, but it does not provide a cure.

- Follow-up must be carried out over long periods and the time interval between initial treatment and metastasis occurrence has been reported to be as long as 13 years [Michel et al.: J Clin Endocrinol Metab 2013;98:4262-4266].

\section{Novel Insights}

- In skull base tumors, stereotactic radiotherapy has been reported favorably [Capatina et al.: Endocr Relat Cancer 2013;20:291-305].

- Chemotherapy has not demonstrated adequate efficacy [Papaspyrou et al.: Laryngoscope 2013; 123:1830-1836]. Somatostatin analogue treatment for tumors positive for its receptor has also not shown an important benefit [Duet et al.: Laryngoscope 2005;115:1381-1384]. Modern antiangiogenic medications may have some promise, but only for paragangliomas of sites other than the head and neck [Joshua et al.: J Clin Endocrinol Metab 2009;94:5-9]. Peptide receptor radionuclide therapy with radiolabeled agents (metaiodobenzylguanidine or somatostatin analogue) is an option for malignant or unresectable paragangliomas with up to half of the cases showing symptomatic and partial tumor response [Capatina et al.: Endocr Relat Cancer 2013;20: 291-305]. Everolimus inhibits mammalian target of rapamycin, a serine-threonine kinase that stimulates cell growth, proliferation, and angiogenesis [Yao et al.: J Clin Oncol 2008;26:43114318]. A phase II study evaluating the effect of everolimus in neuroendocrine tumors demonstrated that everolimus treatment presented with high efficacy and tolerability in patients with nonfunctioning neuroendocrine tumors, but discreet effectiveness in patients with pheochromocytoma/paraganglioma [Oh et al.: Cancer 2012;118:6162-6170].

\section{Introduction}

Paragangliomas originate from pluripotent neural crest stem cells of the autonomic nervous system, known as extra-adrenal paraganglia. Since they may occur anywhere along the paraganglial system, these tumors can be found from the skull base to the sacrum $[1,2]$.

Head and neck paragangliomas derive from the paraganglia of the parasympathetic nerve in the head, neck, and mediastinum [3]. The 4 most common sites of development are the carotid bifurcation, jugular foramen, vagus nerve, and middle ear. Due to the extensive distribution of the autonomic nervous system, they can appear in other locations, such as the orbit, thyroid, larynx, nasopharynx, tongue, and paranasal sinus [1, 4-6]. Despite its cytoplasmic neurosecretory granules, only $1-3 \%$ are functioning paragangliomas with catecholamine secretion [1]. 
From all paraganglioma sites, around 3\% occur in the head and neck, and paragangliomas correspond to less than $0.5 \%$ of all head and neck tumors, with an estimated clinical incidence of $1 / 100,000$ patients per year $[6,7]$. They have a higher predisposition to females, and patients are usually diagnosed in their fifth to seventh decade, and pediatric cases suggest genetic predisposition $[1,5]$. Hereditary paraganglioma incidence is between 10 and $50 \%$, which is often multicentric or bilateral, and is more than a decade younger $[8,9]$. A positive family history increases the risk of multifocality in up to $78 \%$ of the cases [9].

Paraganglionic tissue in the nasal cavity of adults has never been reported. The presence of these cells has been reported in stillborn infants, and the origin of paragangliomas of this site has been suggested to derive from residual specialized neural crest cells along cranial arteries [10-12].

Sinonasal paragangliomas are infrequent, with only approximately 40 cases described [5]. Patients often complain about recurrent epistaxis, nasal obstruction, facial swelling, frontal headache, and loss of olfaction $[4,13]$. These tumors must be differentiated from other sinonasal masses, benign and malignant, such as esthesioneuroblastomas, gliomas, angiofibromas, hemangiopericytomas, rhabdomyosarcomas, and metastases from neuroendocrine tumors [14]. They most commonly arise from the ethmoid sinus, followed by lateral nasal wall and middle turbinate, and are slow-growing tumors, tending to present in older patients [5]. Sinonasal paragangliomas are most often diagnosed in middle-aged women with no personal or family history of paragangliomas. The average age at presentation is 45 years, and most patients are between 40 and 60 years old $[5,14,15]$. Only 3 cases of functioning sinonasal paragangliomas have been described: the first presented as Cushing syndrome and had elevated ACTH levels; the second had a late onset of Cushing's syndrome, presenting 10 years after the first resection; and the last was a catecholamine-secreting tumor in a patient with previous diagnosis of abdominal paragangliomas [16-18].

Most head and neck paragangliomas are benign and have slow progression [2]. Their histological features are not accepted as definitions of malignancy criteria since there is not much difference between benign and malignant paragangliomas. Malignant paragangliomas are defined by lymph node or distant metastasis and may occur in about $10 \%$ of patients [1,2].

Genetic analyses disclosed the role of mutations in the succinate dehydrogenase (SDH) genes in familial and nonfamilial paragangliomas [19]. Succinate dehydrogenase enzymatic activity is lost partially or completely in the tumor, despite the typo of gene mutation 8. Five gene loci with autosomal dominant transmission have been identified for familial paragangliomas: PGL-1, PGL-2, PGL-3, PGL-4, and PGL-5 [3, 9].

Sinonasal paragangliomas are associated with nasal epistaxis, obstruction, facial swelling, pain, and hyposmia [5]. Clinically, they usually are seen as a reddish polypoid mass fixed to the lateral wall of nasal fossa or on the upper area of nasopharyngeal roof and (sometimes) extending to the paranasal sinuses [10, 20-31].

Despite rarely being secretory, all head and neck paragangliomas should have plasma or 24-h urinary metanephrine or catecholamine concentrations measured [32]. If catecholamine excess is present, synchronous pheochromocytoma or other functional paragangliomas must be assessed [33].

Pediatric paragangliomas insinuate genetic predisposition [4] and screening for multiple germline mutations can be performed, such as SDHB and SDHD, which are the most important for predicting a malignant nature $[14,19]$.

Radiologic investigation is essential for diagnosing sinonasal paragangliomas and evaluates their extension and possible skull base invasion. Angiography is important for defining vascular anatomy preoperatively; demonstrating tumor blood supply, bilateral cerebral blood supply, and possible vessel invasion; and ensuring preoperative embolization, which can reduce intraoperative bleeding [34-36]. 
Nuclear imaging may also be required, especially in genetic cases, to delineate the extent of the tumor [2] and detect possible synchronous lesions in mutation carries or those at risk (positive family history and young age) [9]. Metaiodobenzylguanidine ( ${ }^{123}$ I-MIBG), despite its high specificity, has low sensitivity for head and neck paragangliomas, and somatostatin receptor scintigraphy has better sensitivity $[37,38]$.

Due to its higher availability and lower cost, computed tomography (CT) is usually the first exam realized and it performs a better delineation of osseous structures when compared to magnetic resonance imaging (MRI). On the other hand, MRI is superior for identifying a soft tissue's profile and extension [39].

On it histological features, paragangliomas have a typical architecture with the individual tumor cells arranged in nests divided by a fibrovascular stroma, also known as a "Zellballen" pattern $[1,6]$.

\section{Materials and Methods}

This article provides a review of sinonasal paraganglioma, as well as reports an illustrative case of sinonasal paraganglioma and compares it to other similar cases described in the literature. This study was approved by the Brazilian National Cancer Institute and all participants signed an informed consent agreement.

\section{Case Report}

A 16-year-old female presented at the Brazilian Nacional Cancer Institute with a history of spontaneous discrete bilateral epistaxis once a month beginning when she was 3 years old. When she was 14 years old, she started to present with daily profuse bleeding, with no preceding trauma. She had no significant personal or family pathological history.

On physical exam, she had normal vital signs and cranial nerve function. On anterior rhinoscopy, she had a mass in the right nasal cavity. An endoscopy with general anesthesia was performed and a mass occupying the right nasal cavity with extension to the nasopharynx, deviating the septum to the left, was observed.

CT showed an expansive hypervascular mass with well-defined limits occupying the right nasal cavity and nasopharynx. It had nasal septum remodeling and obliteration of maxillary, frontal, sphenoidal, and ethmoidal drainage ostium cells. On MRI, a bulky lobulated mass with well-defined limits and a heterogeneous signal, mainly isointense in T2 and slightly hyperintense in T1 and FLAIR, with intense contrast enhancement and flow-voids in all sequences, was observed. This hypervascular formation filled the right nasal cavity with posterior extension to the nasopharynx and its fibrous palate surface. No other masses were observed in abdominal ultrasonography and thorax CT (Fig. 1, 2). Catecholamine and 24-h urinary metanephrine concentrations were normal, and screening for germline mutations in PGL-1, PGL-2, PGL-3, PGL4, and PGL-5 were negative.

Endonasal biopsy revealed a typical Zellballen pattern and immunohistochemical staining was positive for synaptophysin with a proliferation index (Ki-67) of 5\% and negative for AE1/ AE3, S100, GFAP, CD56, and chromogranin.

Arteriography was performed and a hypervascular lesion nourished by internal maxillary artery and distal branches of the ophthalmic artery bilaterally was observed (Fig. 3, 4).

On the day before surgery, she was submitted to arterial embolization with Embozene 500-700 ${ }^{\circledR}$ (CeloNova BioSciences Inc., San Antonio, TX, USA) particles of internal maxillary and ascending palatine arteries, with important decrement of tumor vascularization. None- 


\section{Biomedicine

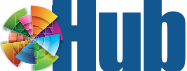

Fig. 1. Sagittal MRI shows the mass filling the nasal cavity with extension to the nasopharynx and its fibrous palate surface.

Fig. 2. Axial MRI shows a bulky well-defined mass filling the nasal cavity.

\begin{tabular}{l}
\hline Biomed Hub 2017;2:464099 (DOI: 10.1159/000464099) \\
\hline (o) 2017 The Author(s). Published by S. Karger AG, Basel \\
www.karger.com/bmh
\end{tabular}

de Almeida Vital et al.: Nasal Cavity Paraganglioma
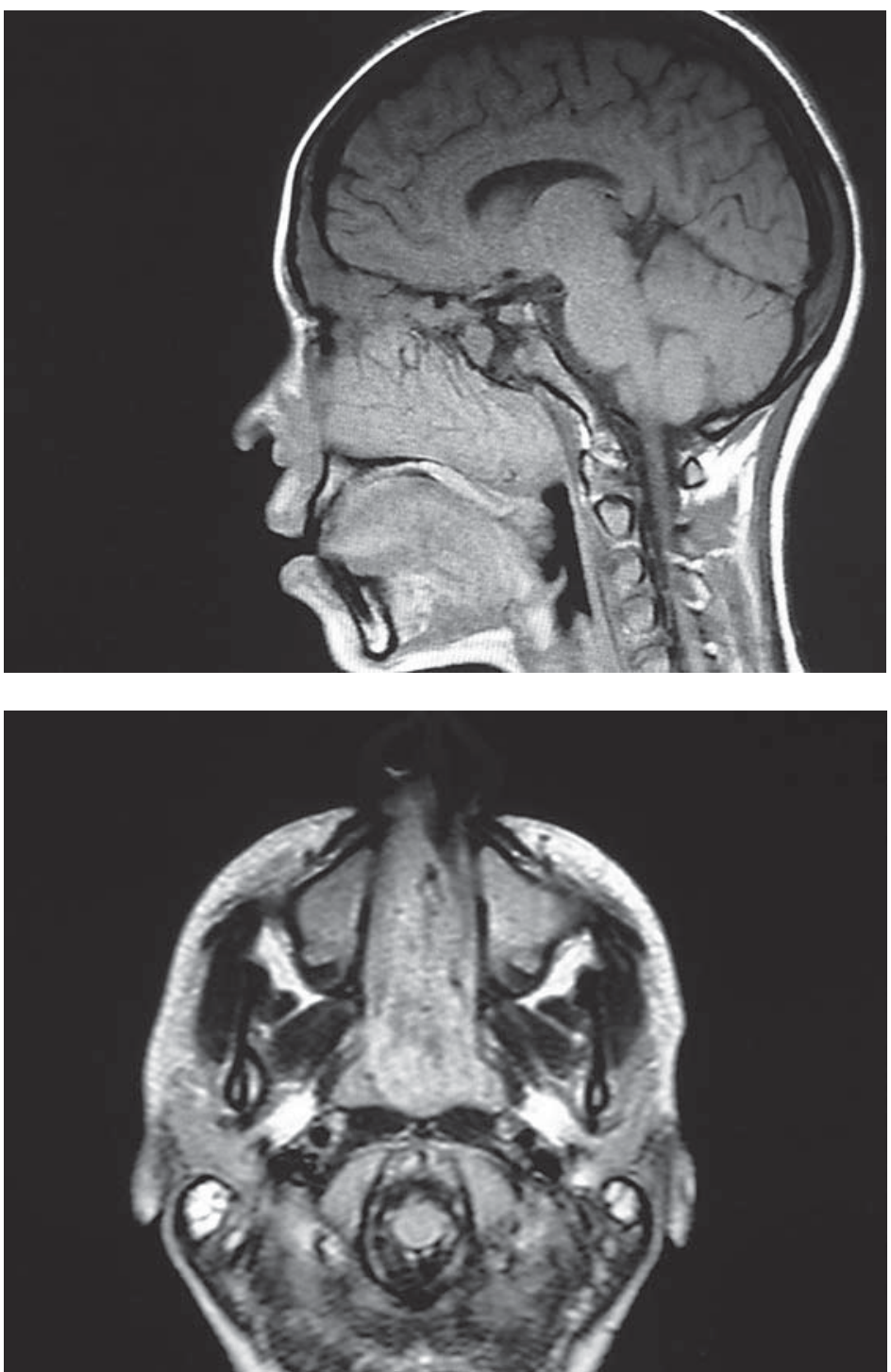

theless, there was residual vascularization from the distal branches of the ophthalmic and superficial temporal arteries.

The complete tumor resection was performed by the Weber-Ferguson and Denker approaches, with free margins confirmed by frozen section. There was no significant bleeding or blood pressure fluctuation during the procedure. Throughout the postoperative setting, the patient had no bleeding or other complications, and the patient was discharged in good clinical condition (Fig. 5-8).

\section{Discussion}

Paragangliomas are neuroendocrine tumors originating from pluripotent neural crest stem cells of the autonomic nervous system, known as extra-adrenal paraganglia, which can emerge from the skull base to the sacrum $[1,2]$.

Sinonasal paragangliomas are a rare entity which occurs most commonly in middle-aged women, with a median age of 45 years. They are often described in patients 40-60 years of 
Fig. 3. Angiography showing tumor blush and blood supply from the ophthalmic artery.

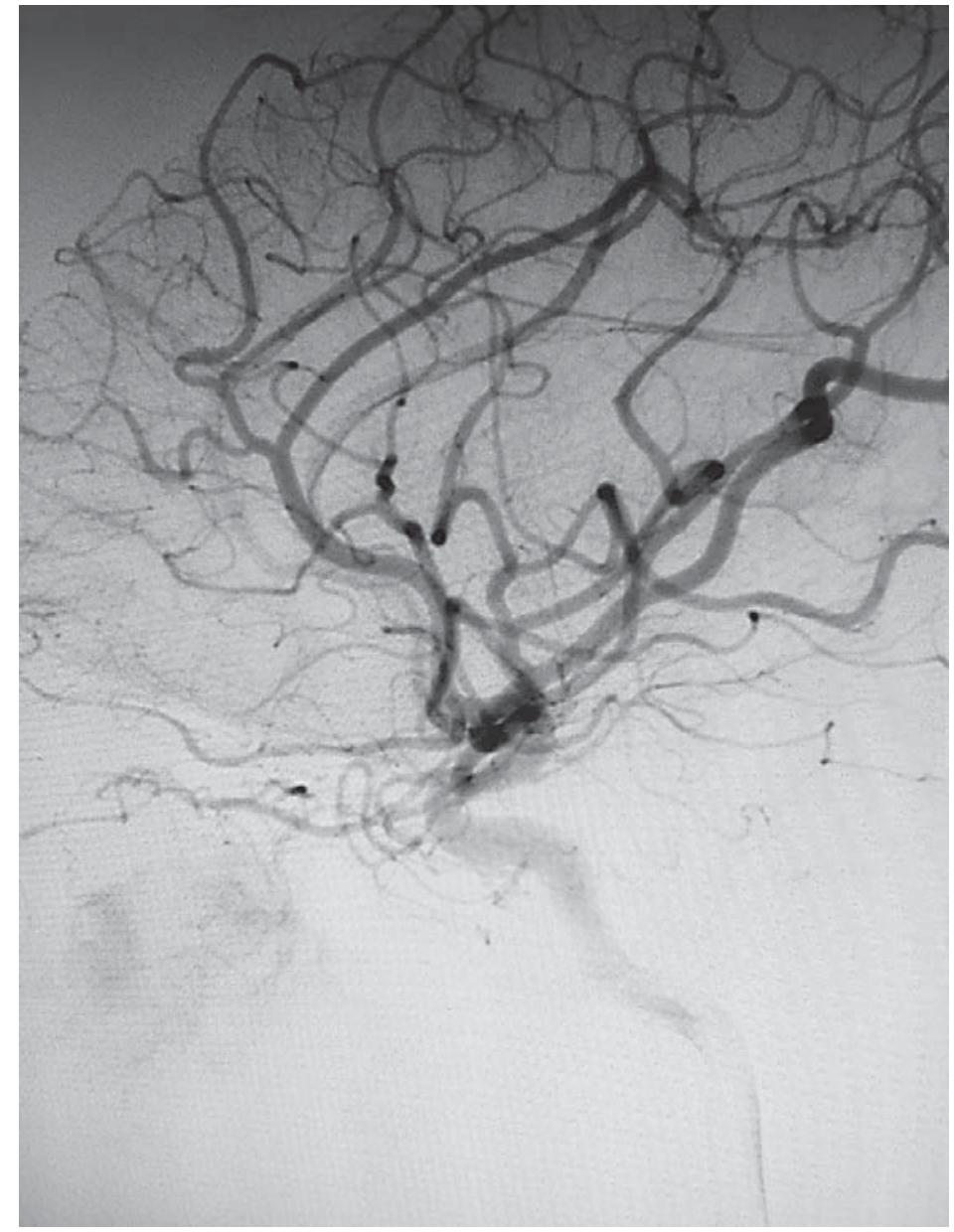

age, but cases have been reported to range in age from 8 to 89 years [1, 5, 34]. Our patient was 16 years old, the second youngest age of onset reported.

Hereditary paraganglioma-pheochromocytoma syndromes comprise PGL1, PGL2, PGL3, PGL4, and PGL5. PGL1 is associated with pheochromocytoma or head and neck paragangliomas, gastrointestinal stromal tumors, and pituitary adenoma, and relates to mutation in the SDHD gene. PGL2 relates to mutation in the SDHAF2 gene and presents as head and neck paraganglioma without other known lesions. PGL3 is related to mutation in the SDHC gene and is associated with head and neck paragangliomas and gastrointestinal stromal tumors. PGL4 relates to mutation in the SDHB gene and the disease could present as pheochromocytoma or head and neck paraganglioma, gastrointestinal stromal tumors, and renal cell carcinoma. PGL5 correlates with mutation in the SDHD gene and could present with paraganglioma and gastrointestinal stromal tumors [3].

Carney-Stratakis syndrome, or Carney dyad, is autosomal dominant and associated with mutations in the SDHB, SDHC, and SDHD genes. It is characterized by paragangliomas and gastrointestinal stromal tumors. Carney's triad is associated with a combination of 3 rare tumors, gastrointestinal stromal tumors, paraganglioma, and pulmonary chondroma, and adrenal cortical adenoma and esophageal leiomyoma may be present [3].

Head and neck paragangliomas have also been associated with Hippel-Lindau syndrome, which presents with hemangioblastoma, retina adenomas, and renal cancer, and are associated with VHL gene mutation [8]. 


\section{Biomenticicine 紏四的}

Fig. 4. Angiography showing tumor blush and blood supply from the internal maxillary artery.

Fig. 5. Surgical field demonstrating a Weber-Ferguson approach for the upper cheek flap with delineation of the Denker approach.
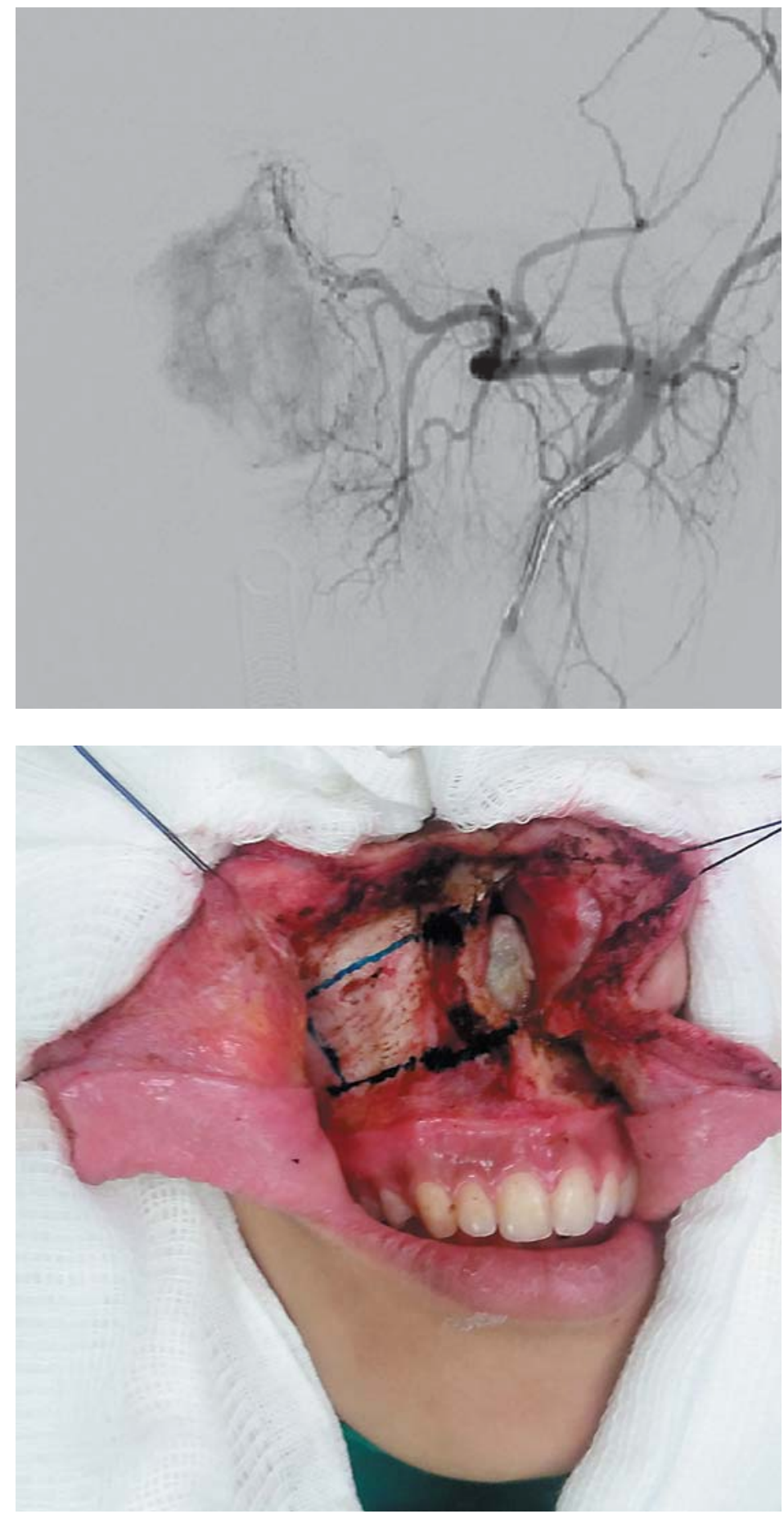

It has been suggested that genetic testing be proposed to all patients undergoing surgery, guided by family history and clinical findings. The first mutation tested should be guided by clinical presentation. If the first is negative, genetic testing should be continued by screening other susceptibility genes [8]. Genetic testing was performed in our patient, but, despite the early onset, genetic mutations or other clinical findings compatible with any genetic syndrome were not observed. 


\section{-

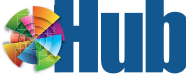

Fig. 6. Surgical field demonstrating the tumor partially excised, fixed by its pedicle located at the roof of nasopharynx.

Fig. 7. Tumor specimen totally excised en bloc.
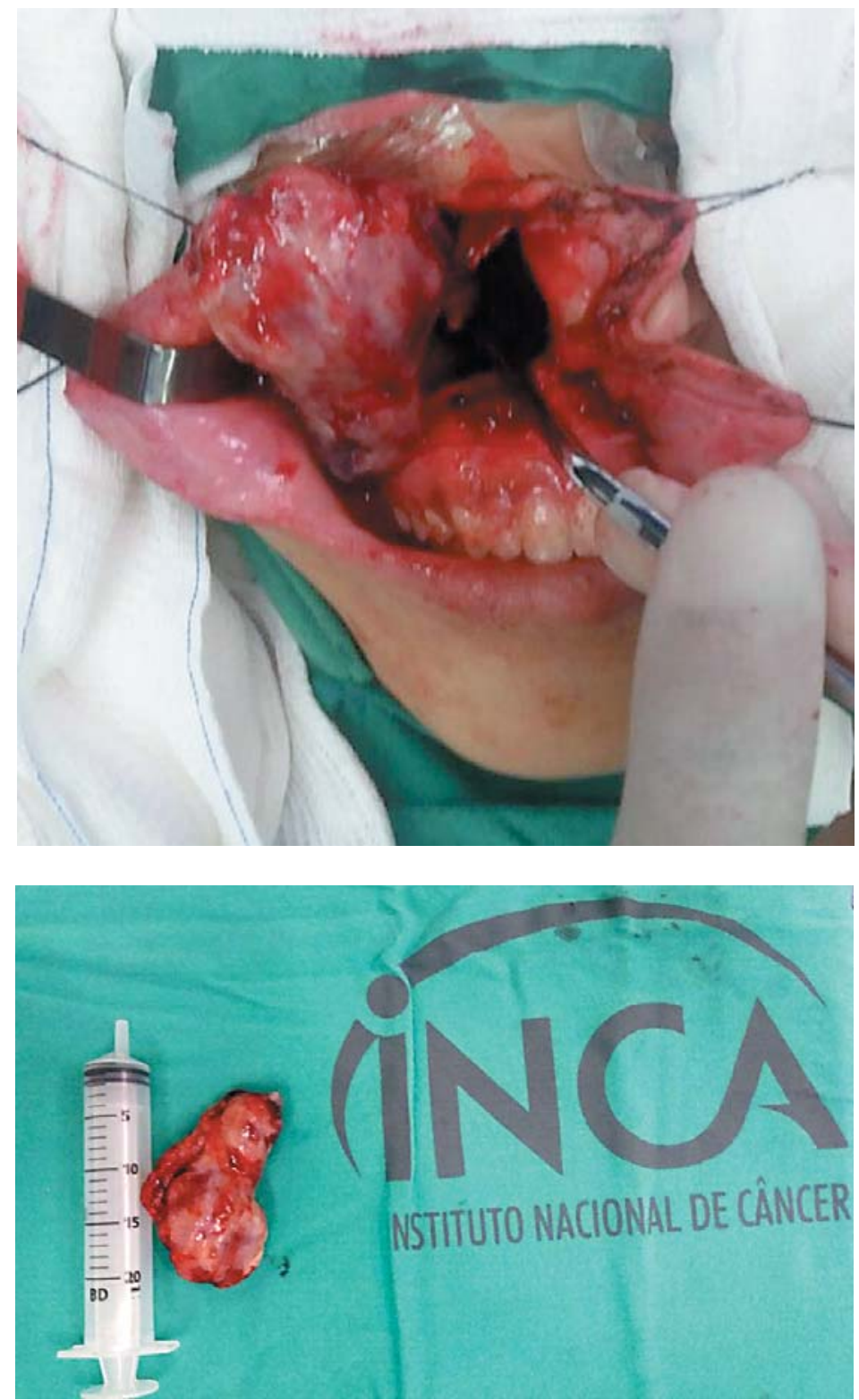

Clinical presentation of these tumors resembles any expansible nasal mass lesion [1]. The most common complaint made by patients with sinonasal paragangliomas are epistaxis and nasal congestion. Despite our patient's tumor size, her main symptom was epistaxis. The youngest patient diagnosed with sinonasal paraganglioma ever reported was an 8-year-old boy with swelling of the left side of the face and a mass in the left turbinate [20]. After him, our patient is the second youngest patient and the youngest female, with 16 years at diagnosis; however, she had a suggestive complaint for the previous 13 years. The age of diagnosis is usually between the fifth and seventh decades [34].

CT and MRI are both useful for sinonasal paraganglioma evaluation, and provide complementary information. Regarding bone invasion, CT has better performance and can also show calcifications, although MRI is better for exposing soft tissues and its features, such as cellularity profile. Furthermore, MRI demonstrates the tumor's precise location, size, and exten- 
Fig. 8. Appearance of the surgical field after resection and hemostasis showing the lateral maxillary sinus wall and the roof of the nasopharynx.

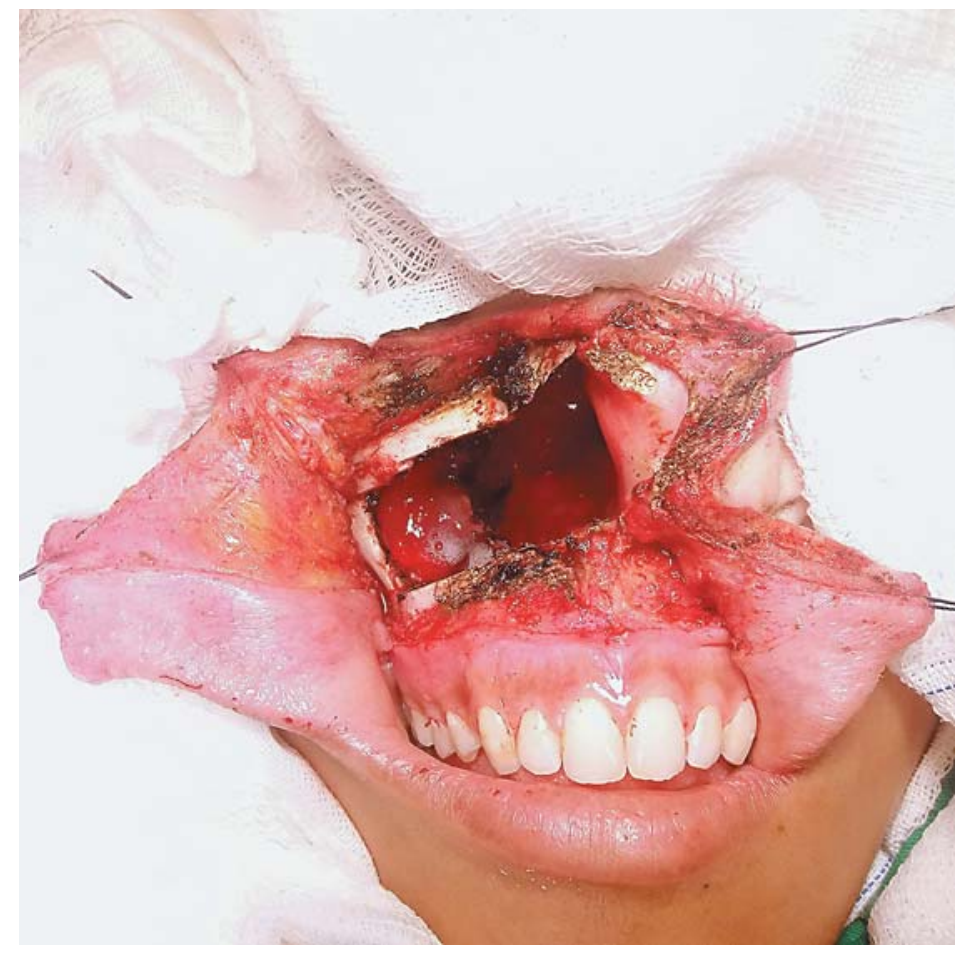

sion to adjacent anatomical sites [40]. The "salt-and-pepper" appearance on MRI is of high diagnostic value, with the "salt" images presenting as high signal areas, secondary to subacute hemorrhage, while the "pepper" areas are low signal intensity flow-voids or vascular channels [41]. The bone remodeling observed on some of these patients is due to the tumor's slow growth pattern. Some these features were observed in our case and others have also reported them.

Most case reports describe expansive masses with well-described limits occupying the nasal cavity with a broad spectrum of extension to adjacent sites, but with a bone remodeling pattern. These are all hypervascular tumors, with contrast enhancement, presenting the classic "salt-and-pepper" appearance and variable patterns of intensity on MRI [5, 10, 20-32].

On CT, our patient presented an expansive hypervascular mass with well-defined limits occupying the right nasal cavity and nasopharynx; nasal septum remodeling; and obliteration of the maxillary, frontal, sphenoidal, and ethmoidal drainage ostium cells. On MRI, a bulky lobulated mass with well-defined limits was observed with a heterogeneous signal, mainly isointense in T2 and slightly hyperintense in T1 and FLAIR, with intense contrast enhancement and flow-voids in all sequences. Those findings are compatible with the different cases reported.

Nuclear imaging may also be required to delineate the extent of the disease [2], as it can detect possible synchronous lesions in confirmed mutation carriers or those at risk (young age and positive family history) [9]. Metaiodobenzylguanidine ( ${ }^{123}$ I-MIBG) is taken by adrenergic tissues, while pentetreotide binds to the somatostatin receptor; these are also known as SPECT scintigraphy [2]. The first, despite its high specificity, has a low sensitivity for head and neck paragangliomas, and somatostatin receptor scintigraphy has better sensitivity [37, 38]. PET imaging sensitivity and resolution are superior to those of SPECT scintigraphy, and it is also a quantitative technique. ${ }^{18} \mathrm{~F}-\mathrm{FDG}$ is the most accessible tracer and it reflects glucose uptake and energy metabolism [2]. Our patient presented with a sporadic case of nonfunc- 
tioning paraganglioma, and since other imaging studies did not demonstrate other tumors, nuclear imaging exams were not necessary.

Differential diagnosis of sinonasal paragangliomas on imaging comprises a broad spectrum of malignant and benign lesions [9], such as esthesioneuroblastomas, gliomas, angiofibromas, hemangiopericytomas, rhabdomyosarcomas, and metastases from neuroendocrine tumors [14].

Esthesioneuroblastoma tumors present as homogeneously enhanced with a dumbbell shape, bone remodeling, and erosion, typically in the midline, centered around the cribriform plate, and are characterized by their peritumoral cyst at the intracranial brain-tumor margin [5].

Gliomas, encephalocele, and dermoid cysts are the most common congenital midline nasal masses, and their lipomatous content is best visualized under MRI. Angiofibroma presents with pathognomonic diffuse-homogeneous and dense contrast enhancement without a necrotic or cystic degeneration mass involving the pterygopalatine fossa and posterior nasal cavity, originating from the sphenopalatine foramen [41].

Juvenile nasopharyngeal angiofibroma is a highly vascular tumor, nonencapsulated, and composed of an irregular network of blood vessels and fibroblastic stroma. It almost exclusively occurs in males, especially adolescents, and presents as a painless nasal obstruction, epistaxis, and nasopharyngeal mass [42]. Its occurrence in females is so rare that some believe it might be a case of pseudohermaphroditism and sex chromosomes studies should be carried out [43]. Androgen receptors are present in at least $75 \%$ of tumors, and some might have progesterone receptors, while estrogen receptors have not been detected [44].

Head and neck rhabdomyosarcoma arises from the orbits and nasopharynx most commonly, usually in children, and $12 \%$ involve the sinonasal cavities. This presents as a nonhomogeneous soft tissue mass with bone erosion, necrosis, and early-stage intracranial growth [5]. Other highly vascular lesions are hemangioma (exceedingly rare in the nasal cavity and images may show a honey-comb appearance) and hemangiopericytomas (approximately $2.5 \%$ of all vascular sinonasal tumors, mostly heterogeneous, with cystic areas and signal-void vessels) $[5,42,45,46]$.

Nuclear imaging may also be required to delineate the extent of the disease [2], detecting possible synchronous lesions in confirmed mutation carries or those at risk (young age and positive family history) [9]. Metaiodobenzylguanidine $\left({ }^{123} \mathrm{I}-\mathrm{MIBG}\right)$ is taken by adrenergic tissues, while pentetreotide binds to somatostatin receptor; these are also known as SPECT scintigraphy [2]. The first, despite its high specificity, has low sensitivity for head and neck paragangliomas, and somatostatin receptor scintigraphy has better sensitivity [37, 38]. PET imaging sensitivity and resolution are superior to those of SPECT scintigraphy, and it is also a quantitative technique. ${ }^{18} \mathrm{~F}-\mathrm{FDG}$ is the most accessible tracer and it reflects glucose uptake and energy metabolism [2]. Our patient presented with a sporadic case of nonfunctioning paraganglioma and other imaging studies did not demonstrate other tumors; nuclear imaging exams were not necessary.

For tumors larger than $3 \mathrm{~cm}$, preoperative hyperselective embolization is advised, preventing major blood loss during surgery [47]. Despite this recommendation, most cases described have not reported embolization previous to the resection.

Several embolic agents have been described and the selection of the embolic material depends on the target, size of the feeding arteries, blood flow pattern, and potentially threatening anastomoses or collateral vessels. Embolic agents might be permanent or temporary. Nonabsorbable materials achieve more durable obstruction and are well suited for vascular lesions [48]. Particulate embolics are the most frequently used particulate embolic agents in modern treatment of head and neck pathology. They have a wide range of diameters, allowing control over depth of vascular penetration. Absorbable gelatin pledgets are not as effective in 
microvascular penetration, as they provide less complete devascularization of tumor beds and the nasal cavity [49].

Our patient was submitted to embolization of the bilateral internal maxillary artery and left ascending palatine artery with Embozene 500-700 ${ }^{\circledR}$ particles, leading to an important reduction of the tumor's vascularization. There was residual vascularization from intracavernous carotid artery branches, mainly from the right distal branches of ophthalmic artery bilaterally, and a distal branch from the right superficial temporal artery. Embozene ${ }^{\circledR}$ particles are spherical and consist of a hydrogel core and apoly(bis[trifluoroethoxy]phosphazene) nanocoat with low inflammatory response [50].

The classic Zellballen pattern is shown in most paragangliomas, with cell nests separated by capillaries and sustentacular cells at its periphery [51]. This pattern was observed in our case, but an uncommon morphology was described with lipoblast-vacuolated cells instead of the typical pattern [23]. Other variations in the tumor architecture include diffuse and trabecular growth patterns, extensive sclerosis that may mimic invasive carcinoma, and unusual vascular patterns that can mimic angiomas [51].

In immunohistochemical staining, neuroendocrine markers such as chromogranin, synaptophysin, and neuron-specific enolase may be positive [52]. Chromogranin seems to be present in most or all paragangliomas, and is thus a useful marker. However, it may fail to stain degranulated cells [22]. Peripheral sustentacular cells demonstrate positivity for S100 protein. Immunohistochemical staining of head and neck paragangliomas can be negative or focally positive for chromogranin A and tyrosine hydroxylase, and sometimes preferably express chromogranin B. The absence of tyrosine hydroxylase explains the usual nonfunctionality of these tumors [51]. Immunohistochemical analysis of our patient's specimen showed positivity only for synaptophysin, and was negative for S100 and chromogranin, which is a compatible pattern for head and neck paraganglioma.

The recognition of malignant and benign paraganglioma by morphologic features is difficult because there is no specific parameter to differ both. However, a tumor's biologic evaluations have shown that malignant paragangliomas show a higher rate of aneuploidy, upregulated expression of CD44 v4/5, which is a marker for an infiltrative growth pattern of a tumor [34].

Sinonasal paragangliomas can show locally disruptive behavior, with recurrence and invasion of sites such as the frontal sinus and orbit [53]. This has been described in over $26 \%$ of metastasis on theses tumors. Additionally, histological features may not be enough to distinguish benign from malignant paraganglioma since there is no particular morphologic character for malignancy [34]. The malignant character of paraganglioma must be defined by the presence of metastasis to cervical lymph nodes or distant organs [1].

In light of the facts explained above, sinonasal paragangliomas must be resected, aiming for free margins. Small tumors can be resected through the endonasal approach, but bleeding control can be difficult, even when preoperative embolization is performed [26]. Based on our patient's tumor extension and vascularity pattern, conventional surgery with preoperative embolization was the treatment of choice. Traditional sinonasal open approaches for sinonasal paraganglioma should be tailored by tumor location and extension. It may be performed by lateral rhinotomy incision, which can be extended with a Weber-Ferguson incision [54]. Lateral rhinotomy provides excellent exposure to the nasal cavity, paranasal sinuses, and nasopharynx with minimal postoperative deformity [55]. Other approaches consist of midfacial degloving, which is suited for extensive lesions in the nasal cavity, ethmoid and sphenoid sinuses, nasopharynx, and infratemporal fossa, avoiding external incision. On the other hand, it may be associated with upward rotation of the nasal tip [56]. The Denker approach, which consists of a wide anterior antrostomy with the removal of the ascending process of the maxilla and the inferior half of the lateral nasal wall, can be associated with the 
previous approach without late complications [57]. The Weber-Ferguson incision combined with the Denker approach was performed, due to its excellent exposure, good aesthetical results, and lack of major complications.

Radiation therapy may control the disease by slowing its growth rate, but it does not provide a cure. Tumor control with radiotherapy is defined as a lack of progression, and complete remission is very rare. The control rate decreases with time. In skull base tumors, stereotactic radiotherapy has been reported favorably. Nevertheless, it has been advocated for unresectable tumors, positive margins after tumor resection, or in cases with aggressive behavior or a histological pattern [9].

Chemotherapy also has not demonstrated adequate efficacy [34]. Somatostatin analogue treatment for tumors positive for its receptor has also not shown an important benefit [58]. Modern antiangiogenic medications may have some promise, but only for paragangliomas of sites other than the head and neck [59]. Peptide receptor radionuclide therapy with radiolabeled agents (metaiodobenzylguanidine or somatostatin analogue) is an option for malignant or unresectable paragangliomas with up to half of the cases showing symptomatic and partial tumor response [9]. Everolimus inhibits mammalian target of rapamycin, a serine-threonine kinase that stimulates cell growth, proliferation, and angiogenesis [60]. A phase II study evaluating the effect of everolimus in neuroendocrine tumors demonstrated that everolimus treatment presented with high efficacy and tolerability in patients with nonfunctioning neuroendocrine tumors, but discreet effectiveness in patients with pheochromocytoma/paraganglioma [61].

Follow-up must be carried out for long periods and the time interval between initial treatment and metastasis occurrence has been reported to be as long as 13 years. There is no gold standard imaging, but CT, MRI, and ${ }^{18} \mathrm{~F}$-FDG PET/CT have been suggested as first-line exams to assess locoregional extension and/or metastatic lesions [14].

\section{Conclusion}

Paragangliomas are infrequent neuroendocrine tumors that may arise from the skull base from the sacrum. Sinonasal paragangliomas are very rare and must be differentiated from other benign and malignant tumors of this site, such as esthesioneuroblastomas, gliomas, angiofibromas, hemangiopericytomas, rhabdomyosarcomas, or metastases from neuroendocrine tumors.

Diagnostic investigation includes catecholamine excess evaluation, CT, and MRI. Nuclear imaging may be required for secondary lesion investigation. Genetic mutations have been described in paraganglioma patients, and those with early onset should be evaluated when genetic testing is available.

Both endoscopic and open conventional approaches are effective in sinonasal paragangliomas, and preoperative embolization is paramount for reducing bleeding risk. Radiotherapy may control the disease by slowing its progression, but cure is unlikely to be achieved. Chemotherapy efficacy has not been demonstrated and peptide receptor radionuclide therapy may be an option in the future for malignant and unresectable paragangliomas.

Histopathological features cannot differentiate benign from malignant paragangliomas, and metastasis may occur at a late phase of the disease. Follow-up must be carried out with thorough imaging investigation for long periods.

The case presented above is the second youngest reported case. It was diagnosed by endoscopic biopsy, CT, and MRI, demonstrating a large nasal cavity paraganglioma. Our patient was submitted to preoperative embolization with particles and open conventional surgery by the Weber-Ferguson and Denker approaches, with total en bloc resection with free margins and an excellent final result, without postoperative complications. 


\section{Acknowledgements}

The authors would like to thank the Research Center of Brazilian National Cancer Institute.

\section{Disclosure Statement}

The authors report no conflicts of interest. This research did not receive any specific grant from funding agencies in the public, commercial, or not-for-profit sectors.

\section{References}

1 Pellitteri P, et al: Paragangliomas of the head and neck. Oral Oncol 2004;40:563-575.

-2 Taïeb D, Neumann H, Rubello D, Al-Nahhas A, Guillet B, Hindié E: Modern nuclear imaging for paragangliomas: beyond SPECT. J Nucl Med 2012;53:264-274.

-3 Lam AK: Update on paragangliomas and pheochromocytomas. Turk Patoloji Derg 2015;31:105-112.

-4 Rao AB, Koeller KK, Adair CF: From the archives of the AFIP. Paragangliomas of the head and neck: radiologicpathologic correlation. Armed Forces Institute of Pathology. Radiographics 1999;19:1605-1632.

-5 Tolman CJ, Stam OCG: Nasal paraganglioma: differential diagnosis from a radiologic and pathologic perspective. BJR Case Reports 2016;2016005.

-6 Offergeld C, Brase C, Yaremchuk S, Mader I, Rischke H, Gläsker S, et al: Head and neck paragangliomas: clinical and molecular genetic classification. Clinics (Sao Paulo) 2012;67:19-28.

7 Baysal BE: Hereditary paraganglioma targets diverse paraganglia. J Med Genet 2002;39:617-622.

$>8$ Burnichon N, Abermil N, Buffet A, Favier J, Gimenez-Roqueplo AP: The genetics of paragangliomas. Eur Ann Otorhinolaryngol Head Neck Dis 2012;129:315-318.

-9 Capatina C, Ntali G, Karavitaki N, Grossman AB: The management of head-and-neck paragangliomas. Endocr Relat Cancer 2013;20:291-305.

10 Lecanu JB, Arkwright S, Halimi PH, Trotoux J, Bonfils P: Multifocal malignant paraganglioma of the paranasal sinuses: a case report. Otolaryngol Head Neck Surg 2002;126:445-447.

11 Rocha MP, Macedo VS, Scarton FB, Rocha HP, Kuhl G: Nasal paraganglioma: a case report. Braz J Otorhinolaryngol 2005;71:237-240.

$\checkmark 12$ Schuller DE, Lucas JG: Nasopharyngeal paraganglioma. Report of a case and review of literature. Arch Otolaryngol Head Neck Surg 1982;108:667-670.

13 Jin HR, Lee OJ, Ahn Y: Nasal cavity paraganglioma with malignant transformation: a case report. Auris Nasus Larynx 2008;35:137-139.

14 Michel J, Taïeb D, Jolibert M, Torrents J, Wassef M, Morange I, et al: Sinonasal paraganglioma with long-delayed recurrence and metastases: genetic and imaging findings. J Clin Endocrinol Metab 2013;98:4262-4266.

15 Said-Al-Naief N, Ojha J: Hereditary paraganglioma of the nasopharynx. Head Neck Pathol 2008;2:272-278.

16 Apple D, Kreines K: Cushing's syndrome due to ectopic ACTH production by a nasal paraganglioma. Am J Med Sci 1982;283:32-35.

17 Lieberum B, Jaspers C, Münzenmaier R: ACTH-producing paraganglioma of the paranasal sinuses. HNO 2003; 51:328-331.

18 Hahn S, Palmer JN, Adappa ND: A catecholamine-secreting skull base sinonasal paraganglioma presenting with labile hypertension in a patient with previously undiagnosed genetic mutation. J Neurol Surg Reports 2012;73:19-24.

19 Baysal BE, Maher ER: 15 years of paraganglioma: genetics and mechanism of pheochromocytoma-paraganglioma syndromes characterized by germline SDHB and SDHD mutations. Endocr Relat Cancer 2015;22:T71T82.

20 Lack E, Cubilla A, Woodruff J, Farr H: Paragangliomas of the head and neck region: a clinical study of 69 patients. Cancer 1977;39:397.

21 Battiata B, Vander W, Adair C, Mair E: Pathology forum: diagnosis: intranasal glomus tumor. Arch Otolaryngol Head Neck Surg 2001;127:329-330.

-22 Amiraraghi N, Syed MI, Syed S, Williams AT: Paraganglioma of the skull base presenting as nasal polyps. Laryngoscope 2013;123:577-580.

23 Aydın S: A case of sinonasal paraganglioma with a different morphology: nine-year follow-up. Kulak Burun Bogaz Ihtis Derg 2015;25:43-45.

24 Fasunla AJ, Ibekwe TS, Afolabi OA, Onakoya PA, Oluwasola OA, Ogun OG, et al: Sinonasal paraganglioma: a case report. Oral Maxillofac Surg 2008;12:93-96.

25 Granato L, Próspero J, Filho D: Nasal paraganglioma: a case report and literature review. Int Arch Otorhinolaryngol 2014;17:92-95. 
26 Ketabchi S, Massi D, Santoro R, Franchi A: Paraganglioma of the nasal cavity: a case report. Eur Arch Otorhinolaryngol 2003;260:336-340.

27 Himelfarb MZ, et al: Paraganglioma of the nasal cavity. Laryngoscope 1983;93:350-352.

28 Kuhn JA, Aronoff BL: Nasal and nasopharyngeal paraganglioma. J Surg Oncol 1989;45:38-45.

29 Paule PN, Valcárcel AS, Ferriñiguez B, Devis IA, Domínguez FR, Rodríguez LMAI: Paranglioma de fosa nasal. Revista Portuguesa Otorrinolaringologia Cirurgia Cérvico-Facial 2008;46:119.

30 Scott M, Brooker DS, Davis RI: Paraganglioma of the nasal cavity. Ulster Med J 2001;70:149-151.

-31 Ueda N, Yoshida A, Fukunishi R: Nonchromaffin paraganglioma in the nose and paranasal sinuses. Acta Pathol Jpn 1985;35:489-495.

-32 Erickson D, Kudva YC, Ebersold MJ, Thompson GB, Grant CS, Van Heerden JA, et al: Benign paragangliomas: clinical presentation and treatment outcomes in 236 patients. J Clin Endocrinol Metab 2001;86:5210-5216.

-33 Eisenhofer G, Goldstein DS, Sullivan P, Csako G, Brouwers FM, Lai EW, Adams KT, Pacak K: Biochemical and clinical manifestations of dopamine-producing paragangliomas: utility of plasma methoxytyramine. J Clin Endocrinol Metab 2005;90:2068-2075.

-34 Papaspyrou K, Welkoborsky HJ, Gouveris H, Mann WJ: Malignant and benign sinonasal paragangliomas. Laryngoscope 2013;123:1830-1836.

-35 van den Berg R, Wasser MN, van Gils AP, van der Mey AG, Hermans J, van Buchem MA: Vascularization of head and neck paragangliomas: comparison of three MR angiographic techniques with digital subtraction angiography. AJNR Am J Neuroradiol 2000;21:162-170.

36 Hu K, Persky MS: Multidisciplinary management of paragangliomas of the head and neck, part 1. Oncology (Williston Park) 2003;17:983-993.

-37 Gimenez-Roqueplo AP, Caumont-Prim A, Houzard C, Hignette C, Hernigou A, Halimi P, Niccoli P, Leboulleux S, Amar L, Borson-Chazot F, et al: Imaging work-up for screening of paraganglioma and pheochromocytoma in SDHx mutation carriers: a multicenter prospective study from the PGL.EVA Investigators. J Clin Endocrinol Metab 2013;98:E162-E173.

-38 Timmers HJLM, Taieb D, Pacak K: Current and future anatomical and functional imaging approaches to pheochromocytoma and paraganglioma. Horm Metab Res 2012;44:367-372.

-39 Koeller KK: Radiologic features of sinonasal tumors. Head Neck Pathol 2016;10:1-12.

40 Valencia MP, Castillo M: Congenital and acquired lesions of the nasal septum: a practical guide for differential diagnosis. Radiographics 2008;28:205-225; quiz 326.

41 Yildirim D: Nasal cavity masses: clinico-radiologic collaborations, differential diagnosis by special clues. Open J Med Imaging 2012;02:10-18.

42 Boghani Z, Husain Q, Kanumuri VV, Khan MN, Sangvhi S, Liu JK, et al: Juvenile nasopharyngeal angiofibroma: a systematic review and comparison of endoscopic, endoscopic-assisted, and open resection in 1,047 cases. Laryngoscope 2013;123:859-869.

43 Mahajan G, Ghate G, Thomas J, Shah P: Nasal angiofibroma - a rare presentation. Webmedcentral Otorhinolaryngol 2013;4:WMC004337.

44 Hwang HC, Mills SE, Patterson K, Gown AM: Expression of androgen receptors in nasopharyngeal angiofibroma: an immunohistochemical study of 24 cases. Mod Pathol 1998;11:1122-1126.

45 Duval M, Hwang E, Kilty SJ: Systematic review of treatment and prognosis of sinonasal hemangiopericytoma. Head Neck 2013;35:1205-1210.

46 Pang H, Yao Z, Ren Y, Liu G, Zhang J, Feng X: Morphologic patterns and imaging features of intracranial hemangiopericytomas: a retrospective analysis. Onco Targets Ther 2015;8:2169.

-47 Faragò G, Castellani C, Ponzi S, Jankovic C, Saginario V, Berardi C, et al: Preoperative embolization of carotid chemodectoma: a technical challenge that can be customized according to angioarchitecture. Illustrative cases. Neuroradiol J 2013;26:678-682.

-48 Turkmani AH, Dannenbaum M, Chen PR: Preoperative tumor embolization; in Edgell RC, Savitz SI, Dalfino J (eds): Neurointervention in the Medical Specialties. New York, Springer, 2015, pp 245-258.

49 Edgell RC, Sinha P, Varvares MA: Neurointervention and the otolaryngologist: head and neck surgeon; in Edgell RC, Savitz SI, Dalfino J (eds): Neurointervention in the Medical Specialties. New York, Springer, 2015, pp 281-302.

50 Stampfl S, Bellemann N, Stampfl U, Sommer CM, Thierjung H, Lopez-Benitez R, et al: Arterial distribution characteristics of Embozene particles and comparison with other spherical embolic agents in the porcine acute embolization model. J Vasc Interv Radiol 2009;20:1597-1607.

51 Tischler AS, De Krijger RR: Pathology of pheochromocytoma and paraganglioma. Endocr Relat Cancer 2015; 22:T123-T133.

52 Mouadeb DA, Chandra RK, Kennedy DW, Feldman M: Sinonasal paraganglioma: endoscopic resection with 4-year follow-up. Head Neck 2003;25:1077-1081.

53 Das D, Kanjilal R, Sen N, Mahanty BC: Nasal paraganglioma with orbital and cranial extension: a rare case report. Ann Neurosci 2010;16:181-183.

54 Fliss DM, Gil Z: Open surgical approaches to the paranasal sinuses; in: Atlas of Surgical Approaches to Paranasal Sinuses and the Skull Base. Berlin, Springer, 2016, pp 109-137.

55 Mertz JS, Pearson BW, Kern EB: Lateral rhinotomy: indications, technique, and review of 226 patients. Arch Otolaryngol 1983;109:235-239. 
(c) 2017 The Author(s). Published by S. Karger AG, Basel www.karger.com/bmh

de Almeida Vital et al.: Nasal Cavity Paraganglioma

56 Howard DJ, Lund VJ: The midfacial degloving approach to sinonasal disease. J Laryngol Otol 1992;106:10591062.

57 Hardillo JAU, Vander Velden LA, Knegt PP: Denker operation is an effective surgical approach in managing juvenile nasopharyngeal angiofibroma. Ann Otol Rhinol Laryngol 2004;113:946-950.

-58 Duet M, Guichard JP, Rizzo N, Boudiaf M, Herman P, Tran Ba Huy P: Are somatostatin analogs therapeutic alternatives in the management of head and neck paragangliomas? Laryngoscope 2005;115:1381-1384.

59 Joshua AM, Ezzat S, Asa SL, Evans A, Broom R, Freeman M, Knox JJ: Rationale and evidence for sunitinib in the treatment of malignant paraganglioma/pheochromocytoma. J Clin Endocrinol Metab 2009;94:5-9.

-60 Yao JC, Phan AT, Chang DZ, Wolff RA, Hess K, Gupta S, et al: Efficacy of RAD001 (everolimus) and octreotide LAR in advanced low- to intermediate-grade neuroendocrine tumors: results of a phase II study. J Clin Oncol 2008;26:4311-4318.

61 Oh DY, Kim TW, Park YS, Shin SJ, Shin SH, Song EK, Bang YJ: Phase 2 study of everolimus monotherapy in patients with nonfunctioning neuroendocrine tumors or pheochromocytomas/paragangliomas. Cancer 2012; 118:6162-6170. 